\title{
Adsorption and self-assembly of aromatic carboxylic acids on Au/electrolyte interfaces
}

\author{
Bo Han · Zhihai Li • Thomas Wandlowski
}

Received: 20 December 2006 /Revised: 27 January 2007 / Accepted: 30 January 2007 / Published online: 22 February 2007

(C) Springer-Verlag 2007

\begin{abstract}
The adsorption and self-assembly of benzoic acid (BA), isophthalic acid (IA), and trimesic acid (TMA) on Au (111) single crystals and on $\mathrm{Au}(111-25 \mathrm{~nm})$ quasi-single crystalline film electrodes have been investigated in $0.1 \mathrm{M}$ $\mathrm{HClO}_{4}$ by combining in situ surface-enhanced infrared reflection absorption spectroscopy (SEIRAS) and scanning tunneling microscopy (STM) with cyclic voltammetry. All three acids are physisorbed on the electrode surface in a planar orientation at negative charge densities. Excursion to positive charge densities (or more positive potentials) causes an orientation change from planar to perpendicular. Chemisorbed structures are formed through the coordination of a deprotonated carboxyl group to the positively charged electrode surface. The three acid molecules assemble in different ordered patterns, which are controlled by $\pi$-stacking (BA) or intermolecular hydrogen bonds between $\mathrm{COOH}$ groups (IA, TMA). A detailed analysis of the potential and time dependencies of the $v_{(\mathrm{C}=\mathrm{O})}, v_{\mathrm{s}(\mathrm{OCO})}$, and $v_{(\mathrm{C}-\mathrm{OH})}$ vibration modes shows that the strength of lateral interactions increases upon chemisorption with an increasing number of $\mathrm{COOH}$ groups in the sequence of $\mathrm{BA}<\mathrm{IA}<\mathrm{TMA}$. The vibration bands shift to higher wavenumbers due to dipole-dipole coupling, Stark tuning, and electron back donation from the electrode to $\mathrm{COO}^{-}$. In addition, an "indirect" electron donation to the $\mathrm{COOH}$ groups takes place via the conjugated molecular skeleton superimposed on the intermolecular hydrogen bonding.
\end{abstract}

\footnotetext{
B. Han $\cdot \mathrm{Z}$. Li $\cdot$ T. Wandlowski $(\triangle)$

Institute of Bio-and Nanosystems (IBN-3)

and Center of Nanoelectronic Systems for Information

Technology (CNI), Research Center Jülich,

52425 Jülich, Germany

e-mail: th.wandlowski@fz-juelich.de
}

Keywords Aromatic carboxylic acid - Adsorption . Self-assembly $\cdot$ Electrochemistry $\cdot$ SEIRAS $\cdot$ STM

\section{Introduction}

Aromatic carboxylic acids, such as benzoic acid (1-benzenecarboxylic acid, BA), isophthalic acid (1,3-benzenedicarboxylic acid, IA), and trimesic acid (1,3,5-benzenetricarboxylic acid, TMA), are composed of a central phenyl ring and peripherical carboxylic groups $(\mathrm{COOH})$. They have been widely used as pattern-controlling units in crystal engineering and supramolecular assemblies such as 1D tapes and ribbons, 2D networks, or 3D bulk crystals [1-4]. These aromatic carboxylic acids and their metal complexes represent prototype building blocks for various molecular nanoarchitectures. Central structure motifs are directional hydrogen bonding and metal ion/ligand coordination [5-14].

Adsorption and self-assembly of several aromatic carboxylic acids and their derivatives were recently studied at electrified solid/liquid interfaces employing electrochemical scanning tunneling microscopy (STM) [15-20]. Here, the electrode potential acts as a universal tuning source to create and to address molecular nanostructures [21, 22].

In situ spectroscopic experiments, especially vibrational spectroscopy, allow one to obtain a direct insight into molecular structures, the interfacial chemical composition, and the interactions within self-assembled adlayers [23, 24]. For example, the adsorption of BA [17, 18, 25, 26], as well as its derivatives such as $p$-nitrobenzoic acid [25], fluorobenzoic acid [19], and terephthalic acid [20], on $\mathrm{Au}, \mathrm{Pt}$, and $\mathrm{Ag}$ electrodes were studied by in situ IR methods including infrared reflection absorption spectroscopy (IRAS) and surface-enhanced infrared reflection absorption spectroscopy (SEIRAS). These acids are typically 
physisorbed at negative charge densities (potentials) with the phenyl ring oriented parallel to the electrode surface, and chemisorbed at positive charge densities (potentials) with the ring being tilted or perpendicularly oriented. This structure transition is often accompanied by the deprotonation of at least one of the $\mathrm{COOH}$ groups. The analysis of band intensity, peak position, and full width at half maximum (FWHM) reveals the changes in adsorbatesubstrate interaction, surface coverage, and long-range order of the adlayer [18, 23, 24].

We have presented a systematic in situ ATR-SEIRAS study on the adsorption of TMA on quasi-single crystalline $\mathrm{Au}(111-25 \mathrm{~nm})$ film electrodes in our previous work [27, 28]. The steady state chemisorbed adlayer structures were investigated with a specific emphasis on the nature of adsorbate-substrate interactions, the role of intermolecular hydrogen bonding, and the co-adsorption of interfacial water. The SEIRAS results support the coordination of one deprotonated carboxyl group to the positively charged electrode surface in a long-range, ordered "ladder-type" network [27].

The present paper extends our previous experiments to a series of aromatic carboxylic acids having the same conjugated $\pi$-electron systems, but different numbers and positions of the $\mathrm{COOH}$ groups. We report on a comparative study of BA, IA, and TMA, combining cyclic voltammetric, EC-STM, and in situ SEIRAS investigations. We focus on the influence of the molecular structure, specifically the number and position of the $\mathrm{COOH}$ groups, on the molecular adsorption and the selfassembled pattern formation.

\section{Experimental}

\section{Electrochemical measurements}

The $\mathrm{Au}(111)$ electrodes were single crystal cylinders of 4-mm height and 4-mm diameter (EC) or discs of 2-mm height and $10-\mathrm{mm}$ diameter (STM). The gold single crystals were flame-annealed in a butane flame or in a hydrogen flame at red heat (ca. $600{ }^{\circ} \mathrm{C}$ ), and then cooled in highpurity argon before mounting into the EC or STM cells [28]. The Au samples employed in the spectro-electrochemical ATR-SEIRAS experiments were prepared by electron beam evaporation of thin gold films (25-nm mass thickness) onto the (111)-plane of a highly refractive Si hemisphere, and subsequent electrochemical annealing by cycling the electrode potential in the double layer region in $0.1 \mathrm{M} \mathrm{HClO}_{4}$ at $50 \mathrm{mV} \mathrm{s}^{-1}$ for ca. $0.5-1 \mathrm{~h}$.

The voltammetric studies were carried out with an AutoLab (PGSTAT-30) or a FHI potentiostat (ELAB200997). A HEKA (PG-310) potentiostat was employed to control and synchronize the electrochemical potential during the in situ SEIRAS measurements. The reference electrode (RE) was either a trapped hydrogen electrode (RHE) or a mercury sulfate electrode (MSE). A large-area platinum spiral was used as counter electrode (CE). All potentials in this paper are quoted with respect to a trapped hydrogen electrode (RHE, ca. $-310 \mathrm{mV}$ vs. SCE).

\section{ATR-SEIRAS setup}

The SEIRAS experiments were carried out employing a vertical spectroelectrochemical cell in a Kretschmann ATR-configuration [23]. The infrared spectra were measured with a Bruker IF66 V/s Fourier transform spectrometer. The spectral resolution was $4 \mathrm{~cm}^{-1}$ in slow-scan and rapid-scan experiments. Non-polarized infrared radiation from a globar source was focused onto the electrodeelectrolyte interface by passing through the back side of a hemispherical Si prism. The angle of incidence was $70^{\circ}$ referring to the surface normal. The spectra are plotted in absorbance units defined as $A=-\log \left(I / I_{\mathrm{o}}\right)$ where $I$ and $I_{\mathrm{o}}$ represent the intensities of the reflected radiation at the actual sample and at the chosen reference potentials, respectively. Further details of the SEIRAS setup and of the spectra acquisition methods were described in our previous publication $[23,27]$.

\section{Electrochemical STM}

The in situ STM experiments were carried out with a Molecular Imaging Pico-SPM using disc-shaped Au(111) single-crystal electrodes. The STM tips were electrochemically etched tungsten tips $(0.25$-mm diameter), coated with polyethylene. Pt wires served as reference and counter electrodes, respectively. All STM images were recorded in constant current mode with tunneling currents ranging between 3 and $200 \mathrm{pA}$. More details of the STM setup were provided elsewhere [28].

\section{Chemicals and electrolyte solutions}

The solutions were prepared with Milli-Q water (18 M $\Omega$ cm, 2-3 ppb TOC), $\mathrm{HClO}_{4}$ (suprapure, Merck), TMA (for synthesis, Lancaster, twice recrystallized in water), benzoic acid (standard for element analysis, Merck), and isophthalic acid (for synthesis, Merck, twice recrystallized in water).

The glassware was cleaned either in a 1:1 mixture of hot $\mathrm{H}_{2} \mathrm{SO}_{4}$ and $\mathrm{HNO}_{3}$, or by soaking in caroic acid, followed by rinsing cycles with Milli-Q water. All electrolytes were deaerated with argon before and during the experiments. The measurements were carried out at room temperature $\left(20 \pm 0.5{ }^{\circ} \mathrm{C}\right)$. 


\section{Results and discussion}

\section{CV and STM}

Figure 1 shows a series of typical current vs. potential curves of BA, IA, and TMA on $\mathrm{Au}(111)$ in $0.1 \mathrm{M} \mathrm{HClO}_{4}$. The first voltammetric trace recorded for a carefully prepared unreconstructed $\mathrm{Au}(111)-(1 \times 1)$ electrode upon scanning the electrode potential from $0.400 \mathrm{~V}$ towards more negative values is represented by the full line. The dashed line shows the steady state cycles, and the dotted trace illustrates the voltammograms of the adsorbate-free supporting electrolyte. The concentrations of the carboxylic acids were chosen between 0.1 and 0.5 of bulk saturation.

One observes two distinct potential regions, II and IV, which are assigned to two-dimensional, long-range ordered physisorbed and chemisorbed adlayers of the respective carboxylic acids [27-29]. The transition is marked by a broad region III. In situ STM experiments reveal that the physisorbed adlayers of BA, IA, and TMA are disordered at $E \leq 0.200 \mathrm{~V}$ (region I) under the experimental conditions chosen in Fig. 1. The corresponding transition $\mathrm{I} \leftrightarrow \mathrm{II}$ is represented by the decreasing capacitive current for BA and TMA, and a pair of well-developed current peaks $\mathrm{P} 1 / \mathrm{P} 1$ ' in the case of IA. The stability range of II at positive potentials is delimited by a rather broad and asymmetric current peak for BA, and a pair of sharp current peaks P2/P2' for IA. A sequence of high-coverage, physisorbed adlayers of planaroriented molecules is observed for TMA at the positive edge of region II and in region III [28]. A disordered chemisorbed adlayer is observed at higher potentials, which finally develops into a two-dimensional, long-range, ordered pattern (region IV [28, 29]). Upon reversing the direction of the potential scan, the current peak P3', which is clearly developed for IA and TMA, indicates the dissolution of the ordered chemisorbed adlayer. Multiple cycling smears out the characteristic transition peaks and regions, such as $\mathrm{P} 1 / \mathrm{P} 1^{\prime}, \mathrm{P} 2 / \mathrm{P} 2$ ', $\mathrm{P} 3 / \mathrm{P} 3$ ', and region III (cf. dashed lines in Fig. 1, [28, 29]).

The insets in Fig. 1 show high-resolution in situ STM images of selected ordered physisorbed and chemisorbed adlayers of BA, IA, and TMA. Flat-lying BA assembles into a square-type pattern of hydrogen-bonded molecules [29]. IA forms hydrogen-bonded zigzag tapes [29, 30]. A hydrogen-bonded honeycomb motif and parallel rows of linear dimers were observed for TMA [28]. These adlayers are stable at an uncharged or slightly negatively charged $\mathrm{Au}$ (111)- $(1 \times 1)$ electrode.

The ordered chemisorbed adlayers, which are stable at positively charged gold electrodes, exhibit characteristic assemblies of parallel single (BA) and double striped (IA, TMA) rows. The chemisorbed aromatic carboxylic acids are assumed to assemble with the phenyl group tilted or
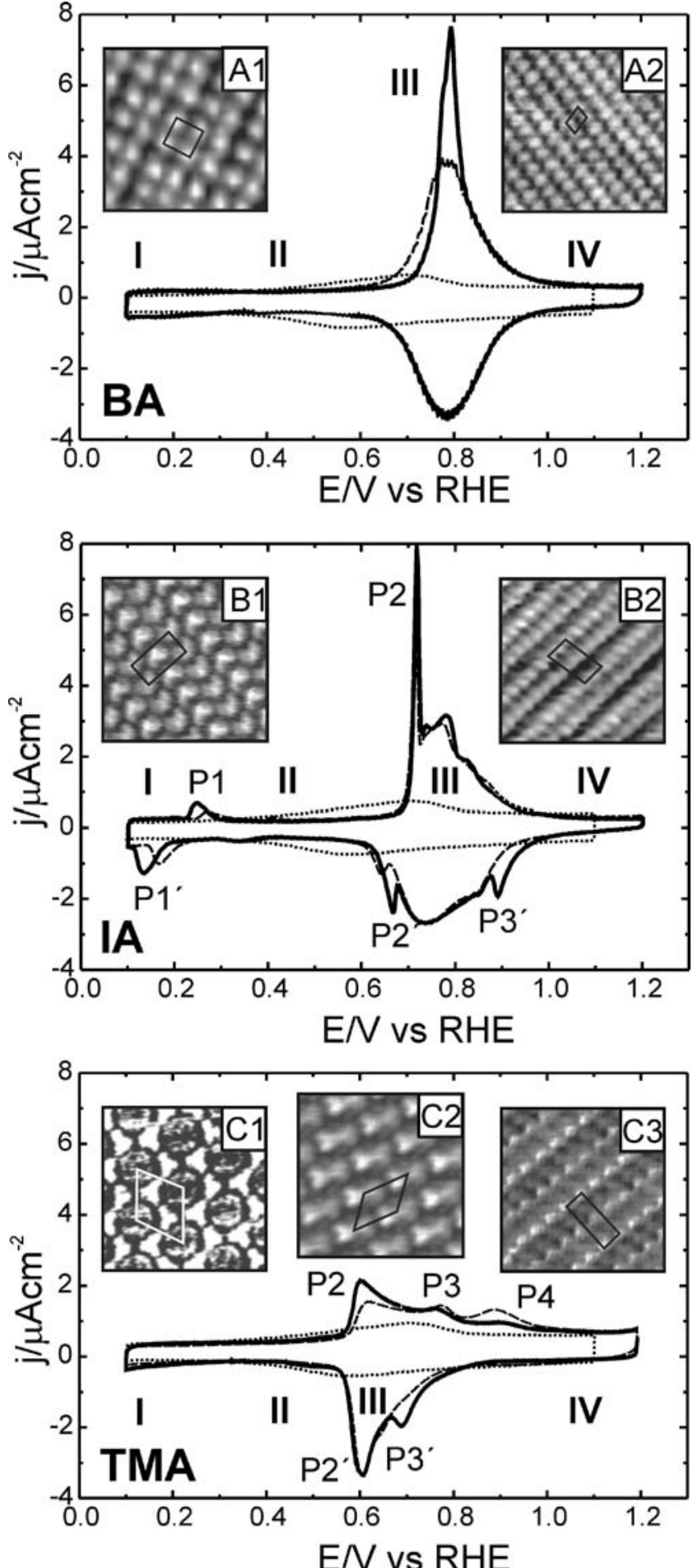

Fig. 1 Cyclic voltammograms (CV) of an ideal $\mathrm{Au}(111)$ electrode (miscut angle $<0.2^{\circ}$ ) in $3 \mathrm{mM} \mathrm{BA} / 0.1 \mathrm{M} \mathrm{HClO}_{4}, 0.5 \mathrm{mM} \mathrm{IA} / 0.1 \mathrm{M}$ $\mathrm{HClO}_{4}$, and $3 \mathrm{mM} \mathrm{TMA} / 0.1 \mathrm{M} \mathrm{HClO}_{4}$ solution, scan rate $10 \mathrm{mV} \mathrm{s}^{-1}$. The solid lines represent the first scan recorded after immersion and stabilization at $0.400 \mathrm{~V}$, the dashed lines show the steady state cycles, and the dotted traces represent the voltammograms in the adsorbatefree supporting electrolyte. The insets represent high-resolution in situ STM images of the ordered phases recorded at $0.65 \mathrm{~V}(A 1), 1.20 \mathrm{~V}$ (A2; BA, $12 \mathrm{mM}), 0.45 \mathrm{~V}(B 1), 1.20 \mathrm{~V}(B 2 ; \mathrm{IA}, 0.5 \mathrm{mM})$, and $0.30 \mathrm{~V}$ $(C 1), 0.70 \mathrm{~V}(C 2), 1.10 \mathrm{~V}(C 3$, TMA, $3 \mathrm{mM})$, respectively. The scale of all STM images is $5 \times 5 \mathrm{~nm}$ 
perpendicularly aligned with respect to the surface normal [27].

The suggested repeat motifs of the ordered adlayers are indicated in the high-resolution STM images. The corresponding dimensions and coverages are summarized in Table 1. Clearly, the surface coverage of the physisorbed and chemisorbed adlayers decrease in the following sequence: $\mathrm{BA}>\mathrm{IA}>\mathrm{TMA}$, which is consistent with the size of the individual molecules. A detailed analysis and discussion of the EC-STM experiments is given elsewhere [28] and [29].

In situ STM experiments and their comparison with crystal structure data reveal clearly that BA, IA, and TMA molecules in the two-dimensional ordered physisorbed state are planar oriented and mutually connected by intermolecular hydrogen bonds between adjacent $\mathrm{COOH}$ groups. On the other hand, the STM data do not allow the resolution of the local molecular structure and the coordination in the chemisorbed state. We therefore employed in situ SEIRAS measurements to investigate the structure details in the chemisorbed adlayers.

\section{In situ SEIRAS}

Figure 2 shows 3D potential-dependent SEIRA spectra of the BA, IA, and TMA adlayers recorded during a slow potential scan with $10 \mathrm{mV} \mathrm{s}^{-1}$. Characteristic vibration bands are observed at positive potentials in the wavenumber range $1,800 \mathrm{~cm}^{-1}>\nu>1,200 \mathrm{~cm}^{-1}$, specifically at $1,720,1,380$, and $1,300 \mathrm{~cm}^{-1}$. The bands around $1,380 \mathrm{~cm}^{-1}$ are attributed to the symmetric stretching $\nu_{\mathrm{s}}$ (OCO) mode of the $\mathrm{COO}^{-}$group $[31,32]$. Considering the low $\mathrm{pH}$ of the electrolyte and the $\mathrm{p} K_{\mathrm{a}}$ values of the three acids (BA: $\mathrm{p} K_{\mathrm{a}}=4.19$; IA: $\mathrm{p} K_{\mathrm{a}}=3.54,4.60$ [33]; TMA: $\mathrm{p} K_{\mathrm{a}}=2.12,4.10$, and 5.18 [34]), we conclude that the observation of the $\nu_{\mathrm{s}(\mathrm{OCO})}$ bands indicates the chemisorption of molecules onto the gold surface via a $\mathrm{COO}^{-}$group. The strong vibration bands at 1,720 (IA), 1,735 (TMA) $\mathrm{cm}^{-1}$, and the weak bands at 1,290 (IA) and 1,320 (TMA) $\mathrm{cm}^{-1}$ are assigned to the stretching modes $\nu_{(\mathrm{C}=\mathrm{O})}$ and $\nu_{(\mathrm{C}-\mathrm{OH})}$ of a protonated $\mathrm{COOH}$ group. Comparing to IRAS data and experiments with smooth $\mathrm{Au}(111)$ electrodes $[17,18,25,26]$ we roughly estimate an enhancement factor for the symmetric stretching mode $\nu_{\mathrm{s}(\mathrm{OCO})}$ of the three carboxylic acids, which ranges between 10 and 50 .

The intensities of all three vibration bands increase during a positive-going potential sweep, and reach their maximum values at $E>1.000 \mathrm{~V}$. The potential dependencies of the vibration bands of BA and IA support the following scenario referring to the surface selection rule of SEIRAS $[35,36]$ and the parallel alignment of the vibration dipoles to the phenyl ring: the excursion to positive potentials causes the breakdown of the physisorbed hydrogen-bonded network, and BA and IA molecules change their orientation from planar to tilted or perpendicular accompanied by the deprotonation of at least one of the carboxyl groups. The latter leads to the formation of a substrate-adsorbate coordination complex. A similar sequence was proposed for TMA on gold [27].

Figure 3 shows selected SEIRA spectra at $E=1.1 \mathrm{~V}$ (region IV), which have been recorded simultaneously with a slow scan $\left(10 \mathrm{mV} \mathrm{s}^{-1}\right)$ voltammogram. The correlation analysis of band intensities, as suggested in our previous work [27], demonstrates that each acid is coordinated via only one $\mathrm{COO}^{-}$group to the positively charged $\mathrm{Au}$ substrate. The remaining $\mathrm{COOH}$ side groups of IA and TMA, which face the electrolyte, contribute to the formation of an intermolecular hydrogen-bonded network. The intensity ratios between the $\nu_{(\mathrm{C}=\mathrm{O})}$ and the $\nu_{(\mathrm{C}-\mathrm{OH})}$ modes of IA and TMA are similar, indicating that both systems have the side $\mathrm{COOH}$ groups with similar orientations in the hydrogen-bonded networks.

Scheme 1 summarizes the structure models of the physisorbed and chemisorbed adlayers of BA, IA, and TMA as derived from the results of the in situ STM and SEIRAS experiments.

Table 1 Details of the STM results for physisorbed (P) and chemisorbed (C) adlayers of BA, IA, and TMA

\begin{tabular}{lllllll}
\hline Molecule & Type of adsorption & Description & Unit cell & & \\
\cline { 3 - 5 } & & & $a(\mathrm{~nm})$ & $b(\mathrm{~nm})$ & $\alpha\left({ }^{\circ}\right)$ & $\left(10^{-10} \mathrm{~mol} \mathrm{~cm}^{-2}\right)$ \\
\hline BA $(12 \mathrm{mM})$ & $\mathrm{P}$ & Linear dimer & $0.79 \pm 0.05$ & $0.81 \pm 0.05$ & $95 \pm 5$ & $2.6 \pm 0.3$ \\
& $\mathrm{C}$ & Single stacking rows & $0.58 \pm 0.05$ & $0.41 \pm 0.05$ & $76 \pm 5$ & $7.4 \pm 0.2^{\mathrm{a}}$ \\
IA $(0.5 \mathrm{mM})$ & $\mathrm{P}$ & Zigzag chains & $0.74 \pm 0.05$ & $1.68 \pm 0.05$ & $90 \pm 5$ & $2.7 \pm 0.3$ \\
& $\mathrm{C}$ & Double stacking rows & $0.80 \pm 0.05$ & $1.55 \pm 0.05$ & $75 \pm 5$ & $5.7 \pm 0.3$ \\
TMA $(3 \mathrm{mM})$ & PI & Hexagonal honeycomb & $1.70 \pm 0.08$ & $1.70 \pm 0.08$ & $60 \pm 6$ & $1.3 \pm 0.2$ \\
& PII & Linear dimer & $1.18 \pm 0.05$ & $1.43 \pm 0.05$ & $63 \pm 5$ & $2.2 \pm 0.1$ \\
& $\mathrm{C}$ & Double stacking rows & $0.86 \pm 0.05$ & $2.15 \pm 0.20$ & $80 \pm 5$ & $3.6 \pm 0.4$ \\
\hline
\end{tabular}

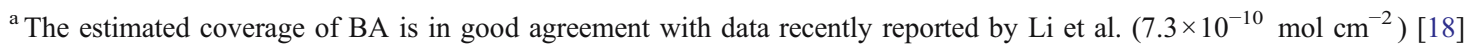




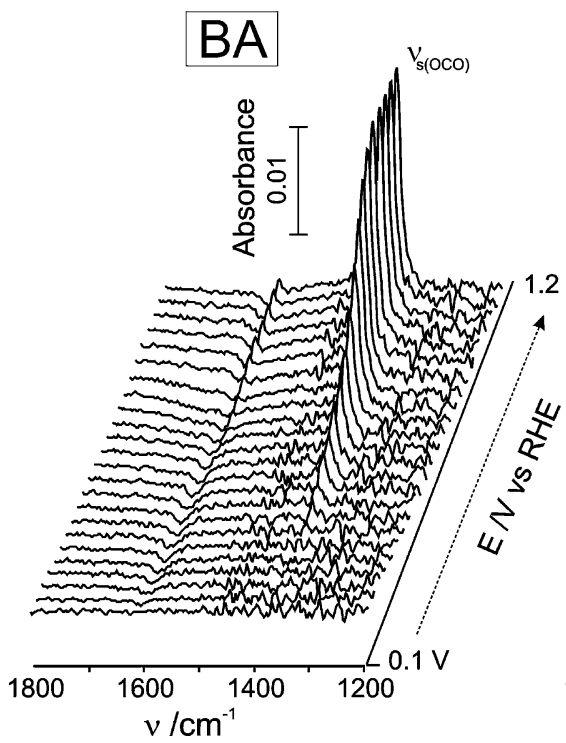

Fig. 2 Typical sets of 3D SEIRA spectra of BA, IA, and TMA adlayers measured simultaneously with the corresponding slow-scan voltammograms $\left(10 \mathrm{mV} \mathrm{s}^{-1}\right)$ during a potential cycle from $E=0.100 \mathrm{~V}$ to $\mathrm{E}=1.200 \mathrm{~V}$ and back to $E=0.100 \mathrm{~V}$. For clarity we only show the

Interactions within adlayers

In situ SEIRAS provides not only direct structure and chemical information of the adlayers, but also fingerprints

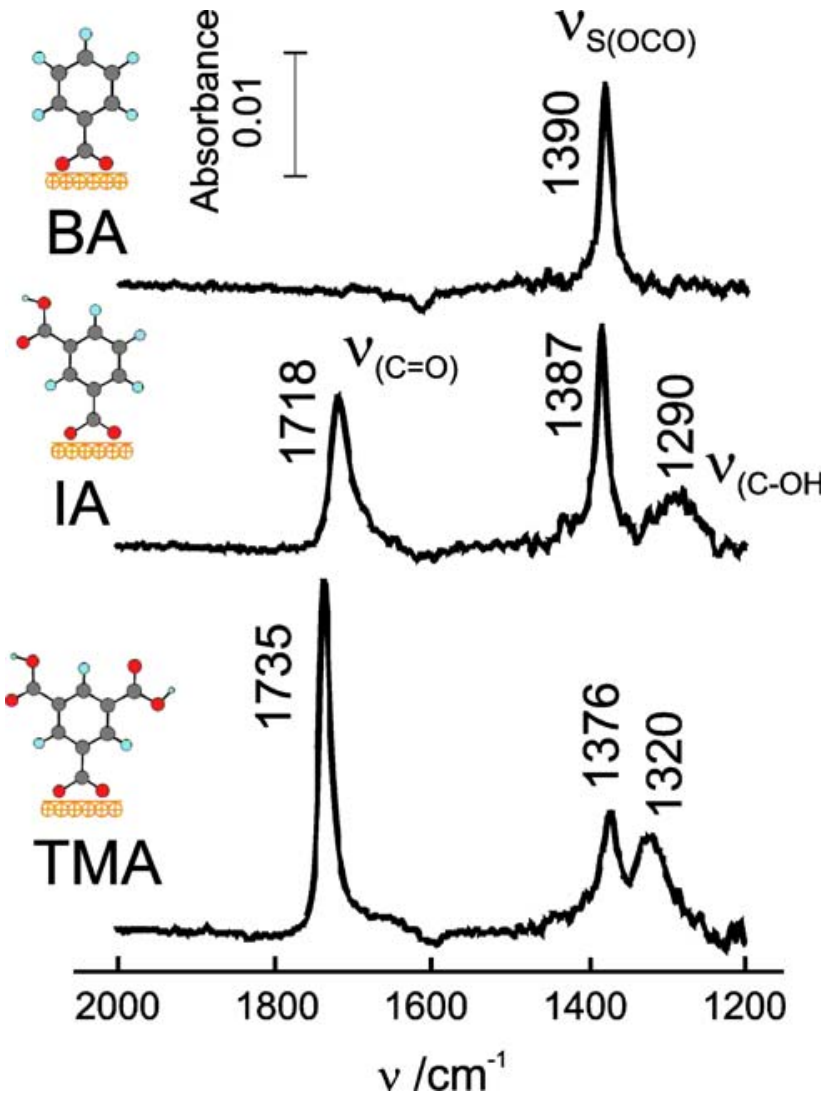

Fig. 3 Selected equilibrium SEIRA spectra of BA, IA, and TMA adlayers obtained at $1.100 \mathrm{~V}$ in region IV (chemisorbed phase). The reference spectrum was recorded at $E=0.100 \mathrm{~V}$

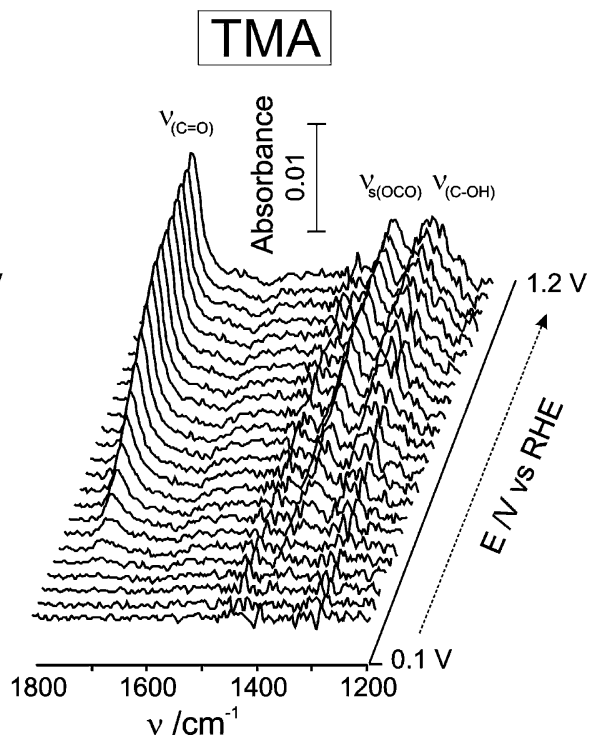

spectra recorded during a positive-going potential sweep. The single beam spectrum acquired at $E=0.100 \mathrm{~V}$ is chosen as reference. Each spectrum represents an average of 160 single traces within a potential interval of $50 \mathrm{mV}$

of the lateral interactions and of the nature of adsorbatesubstrate coupling. This knowledge contributes substantially to the comprehensive understanding of the driving forces for self-assembly. In this contribution we will focus on the IR vibration frequencies of the $\mathrm{COOH}$ and $\mathrm{COO}^{-}$groups, which are the key functional elements for the lateral hydrogen bonding and the adsorbate-substrate coupling in the chemisorbed adlayer phases (region IV).

As shown in Fig. 3, the maximum frequencies of the $\nu_{\mathrm{s}}$ (OCO) bands decrease in the order BA $\left(1,390 \mathrm{~cm}^{-1}\right)>$ IA $\left(1,387 \mathrm{~cm}^{-1}\right)>$ TMA $\left(1,376 \mathrm{~cm}^{-1}\right)$. This sequence indicates that adsorbate-substrate coupling in the long-range ordered chemisorbed adlayers, which occurs via the $\mathrm{COO}^{-}$ groups, is weaker with an increasing number of $\mathrm{COOH}$

\section{Physisorption}

BA

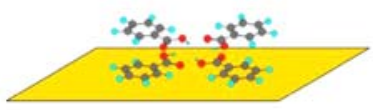

IA
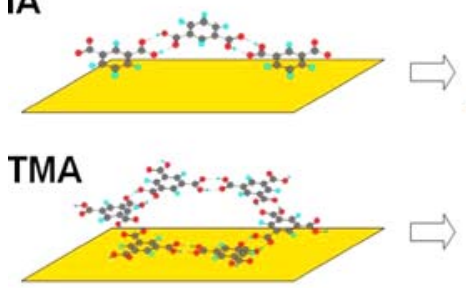

\section{Chemisorption}
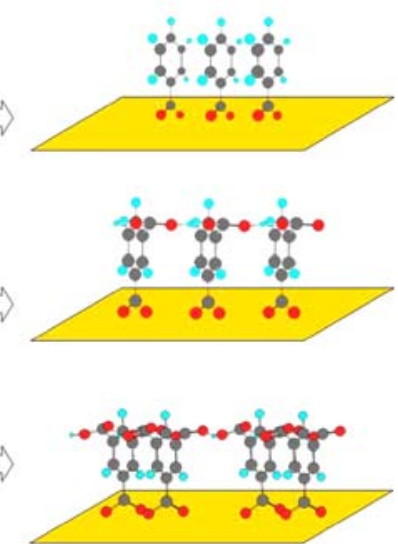

Scheme 1 Models of the steady-state adlayer structures of BA, IA, and TMA in the potential regions II (physisorption) and IV (chemisorption) 
groups. On the other hand, the $\nu_{(\mathrm{C}=\mathrm{O})}$ and $\nu_{(\mathrm{C}-\mathrm{OH})}$ bands of TMA $\left(1,735\right.$ and $\left.1,320 \mathrm{~cm}^{-1}\right)$ appear at higher maximum wavenumbers than those of IA $\left(1,718\right.$ and $\left.1,290 \mathrm{~cm}^{-1}\right)$, which suggests a stronger lateral interaction via the two hydrogen-bonded $\mathrm{COOH}$ groups of each chemisorbed TMA molecule.

The potential (and time) dependencies of the vibration bands provide additional information on the dynamic properties of the transition between the physisorbed and chemisorbed adlayers. The plots of the vibrational frequencies of $\nu_{(\mathrm{C}=\mathrm{O})}, \nu_{(\mathrm{C}-\mathrm{OH})}$, and $\nu_{\mathrm{s}(\mathrm{OCO})}$ versus the potential, as obtained from the spectra plotted in Fig. 2, are presented in Fig. 4. Table 2 summarizes the final band positions estimated at $E=1.200 \mathrm{~V}$ and the corresponding peak shifts. Such potential-dependent peak shifts of vibration modes are generally attributed to three different factors: the Stark tuning effect, metal-adsorbate electron (back) donation, and lateral interactions such as $\pi$-stacking and dipole-dipole coupling [37-39].

\section{Adsorbate-substrate coupling}

The $\nu_{\mathrm{s}}(\mathrm{OCO})$ band of BA shifts to higher frequencies during a positive-going potential sweep. The values of $\nu_{\mathrm{s}}$ (OCO) vs. $E$ increase rather steeply in $0.700 \mathrm{~V}<E<0.950 \mathrm{~V}$. A linear segment with a considerably smaller slope $\left(8.47 \mathrm{~cm}^{-1} \mathrm{~V}^{-1}\right)$ follows at $E>0.950 \mathrm{~V}$. The steep $\nu_{\mathrm{s}}$ (OCO) vs. $E$ dependence is attributed to an increase of the dipole-dipole coupling upon the orientation change and chemisorption of BA [18]. Peak shifts of similar order of magnitude, which were attributed to a strong dipole-dipole coupling, were observed for close-packed chemisorbed adlayers of several organic molecules, such as cytosine [40], 4,4'-bipyridine [41], BA [15, 18, 19], fumaric acid [42], and uracil [24]. The linear part with a rather small slope in the potential range of chemisorption provides a hint of Stark tuning [18], although there is no strong experimental evidence for a predominant Stark tuning effect, i.e., a uniform linear $\nu_{\mathrm{s}(\mathrm{OCO})}$ vs. $E$ dependence in a large potential range [37]. Compared with the strong dipole-dipole coupling of BA, an electron back donation from the $d$-levels of the Au electrode into the $\pi^{*}$-orbital of $\mathrm{COO}^{-}$is less important due to a rather localized $\pi$-electron density in the phenyl ring, and a strong $\sigma$-bonding between the oxygen and $\mathrm{Au}$ atoms [43].

The $\nu_{\mathrm{s}(\mathrm{OCO})}$ band of IA also shifts to higher frequencies during a positive-going potential sweep. However, this blue shift is smaller than that of BA, which is partially attributed to the additional $\mathrm{COOH}$ side group. The latter increases the distance between neighboring $\mathrm{COO}^{-}$groups in the chemisorbed adlayer, and consequently decreases the coupling effect between the dipoles of the $\mathrm{COO}^{-}$anchors. The hysteresis and the clearly developed plateau in the reversed

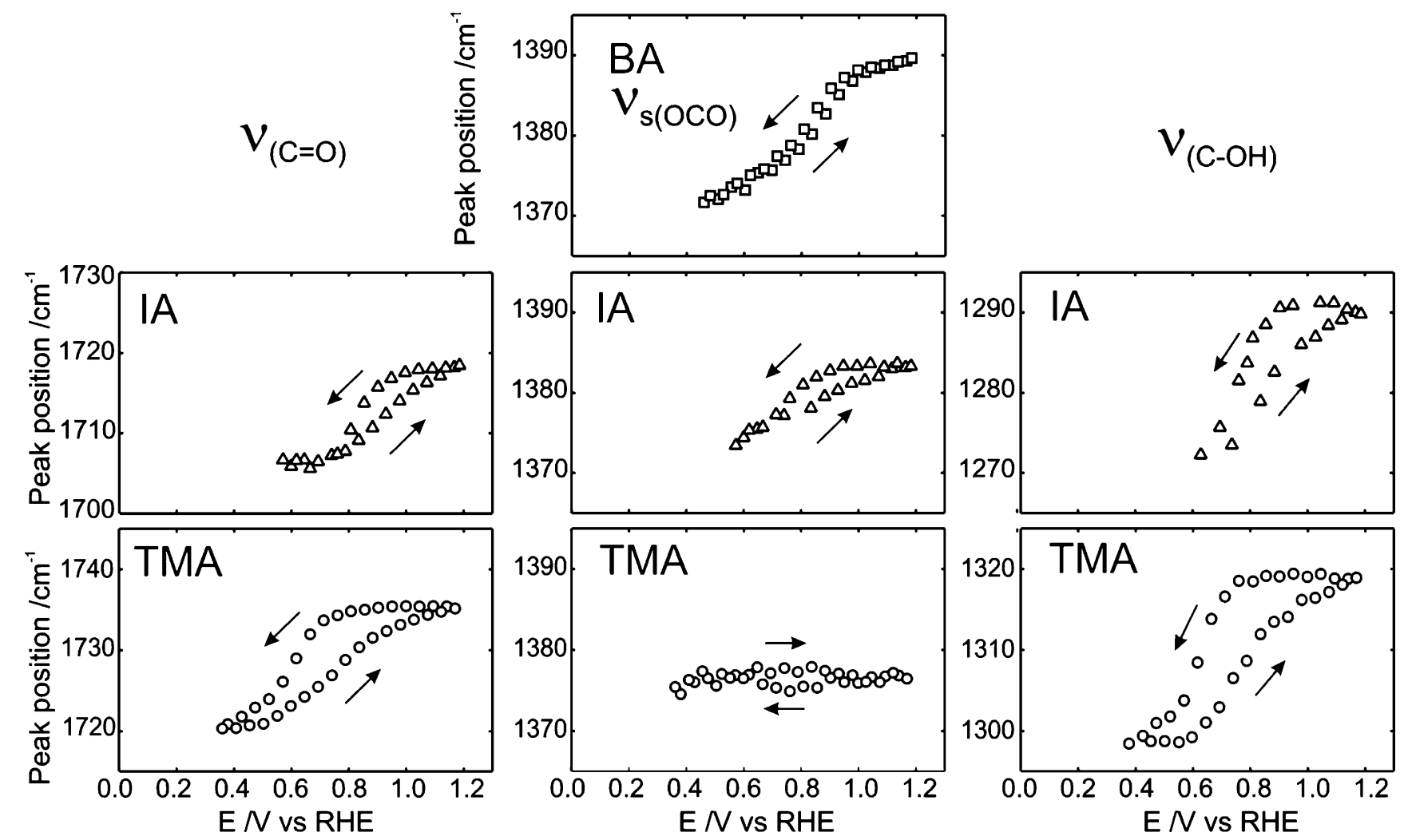

Fig. 4 Potential dependencies of the peak positions of the $\nu_{(\mathrm{C}=\mathrm{O})}, \nu_{\mathrm{s}(\mathrm{OCO})}$, and $\nu_{(\mathrm{C}-\mathrm{OH})}$ bands of BA $(\square)$, IA $(\Delta)$, and TMA $(\circ)$. The data were extracted from Fig. 2 
Table 2 Potential-induced peak shifts $\left(\mathrm{cm}^{-1}\right)$ of the characteristic $\mathrm{COOH}$ and $\mathrm{COO}^{-}$vibrational modes of different carboxylic acids

\begin{tabular}{llllll}
\hline & $\nu_{\mathrm{s}(\mathrm{OCO})}$ & $\Delta \nu_{\mathrm{s}(\mathrm{OCO})}$ & $\nu_{\mathrm{s}(\mathrm{C}=\mathrm{O})}$ & $\Delta \nu_{\mathrm{s}(\mathrm{C}=\mathrm{O})}$ & $\nu_{(\mathrm{C}-\mathrm{OH})}$ \\
\hline BA & 1,390 & 20 & - & - & - \\
IA & 1,387 & 8 & 1,718 & 11 & 1,290 \\
TMA & 1,376 & ca. 0 & 1,735 & 15 & 1,320 \\
\hline
\end{tabular}

The $\Delta \nu$ values were obtained from the subtraction of the spectra at $0.40 \mathrm{~V}$ (BA, TMA) and $0.60 \mathrm{~V}$ (IA) from those at $1.20 \mathrm{~V}$ (cf. Fig. 4 )

negative-going potential sweep, after the $\nu_{\mathrm{s}}(\mathrm{OCO})$ frequency has reached its maximum at $1.200 \mathrm{~V}$, indicate the formation of a rather stable chemisorbed adlayer of IA.

The slope of the $\nu_{\mathrm{s}(\mathrm{OCO})}$ Vs. $E$ plot of TMA is nearly zero. The dipole-dipole coupling within the chemisorbed TMA adlayer is very weak. Neighboring TMA molecules are spatially separated, even further from each other, by two pairs of hydrogen-bonded $\mathrm{COOH}$ groups. This explanation is also supported by the STM results, which clearly demonstrate a decreasing packing density in the sequence $\mathrm{BA}>\mathrm{IA}>\mathrm{TMA}$.

The frequencies of the $\nu_{\mathrm{s}(\mathrm{OCO})}$ mode of IA and TMA are predominantly determined by the Stark tuning effect (blue shift) and the electron back donation (red shift). The dipole-dipole coupling is rather weak. We refer to results of our previous kinetic study in an attempt to distinguish between the two dominant contributions [27]. The establishment of the long-range order within the chemisorbed adlayer due to $\pi$-stacking (BA) or hydrogen bonding (IA and TMA) at a fixed potential was tracked by time-resolved SEIRAS in the rapid scan mode. Figure 5 shows the plots of the vibration frequencies $\nu_{(\mathrm{C}=\mathrm{O})}, \nu_{(\mathrm{C}-\mathrm{OH})}$, and $\nu_{\mathrm{s}(\mathrm{OCO})}$ as a function of time, recorded after a potential step from region II (physisorption) into region IV (chemisorption). Thus, the constant Stark tuning at fixed potentials can be separated from the overall contribution. The plots of IA and TMA in Fig. 5 show only a red shift for the $\nu_{\mathrm{s}(\mathrm{OCO})}$ mode, which is attributed to electron back donation. As derived from Fig. 4, this red shift is partially (IA) or completely (TMA) compensated by the Stark tuning during a positive-going potential sweep. On the contrary, a blue shift is observed in the time dependence of $\nu_{\mathrm{S}(\mathrm{OCO})}$ for BA, which supports the notions that the compact $\pi$-stacking phase of BA (region IV) is dominated by dipole-dipole coupling.

\section{Lateral hydrogen-bonding interaction}

The lateral interactions within an adlayer include intermolecular hydrogen bonding and $\pi-\pi$ stacking between neighboring phenyl planes. The latter may assist to stabilize the compact packing of chemisorbed BA molecules, but is not predominant for IA and TMA having additional $\mathrm{COOH}$ side groups. Therefore, we will focus on the potential dependencies of the $\nu_{(\mathrm{C}=\mathrm{O})}$ and $\nu_{(\mathrm{C}-\mathrm{OH})}$ modes of the $\mathrm{COOH}$ groups, which contribute to the formation of an intermolecular hydrogen-bonded ladder network.
As shown in Fig. 4 , both bands, $\nu_{(\mathrm{C}=\mathrm{O})}$ and $\nu_{(\mathrm{C}-\mathrm{OH})}$, shift for IA and TMA to higher wavenumbers during a positivegoing potential sweep. The most pronounced change occurs also in potential region III, e.g., around the transition between the physisorbed and the chemisorbed state. A plateau develops at positive potentials in the stability region of the chemisorbed phase IV. Upon reversing the direction of the potential scan one observes that the negative edge of the plateau region coincides with $\mathrm{P} 3$ ', the current peak in the voltammograms attributed to the dissolution of the longrange ordered chemisorbed phase. The extension of the plateau region of TMA towards negative potentials $(0.400 \mathrm{~V})$ is larger than that of IA (ca. $0.200-0.300 \mathrm{~V})$, indicating a higher stability of the chemisorbed adlayer of the former. The additional $\mathrm{COOH}$ group of TMA compared to IA may lead to a more compact hydrogen-bonded network, which supports the perpendicularly aligned TMA molecules in region IV (Scheme 1).

Due to the large distance from the substrate, the frequencies $\nu_{(\mathrm{C}=\mathrm{O})}$ and $\nu_{(\mathrm{C}-\mathrm{OH})}$ are hardly influenced by the strong electric field, which dominates in the immediate vicinity of the electrode [39]. In other words, a strong Stark effect is not very probable. Therefore, the blue shifts of the $\nu_{(\mathrm{C}=\mathrm{O})}$ and $\nu_{(\mathrm{C}-\mathrm{OH})}$ bands are dominantly attributed to intermolecular hydrogen bonding and to an "indirect" electron donation to the $\mathrm{COOH}$ side groups via the delocalized $\pi$-electron system in the conjugated molecular skeletons of IA and TMA [27, 28]. Similar red shifts due to the delocalization of $\pi$-electrons have been predicted and observed for terephthalic acid and conjugated nitriles [20, 44]. The above two factors act simultaneously upon the establishment of the chemisorbed adlayers.

The formation of hydrogen bonds between two $\mathrm{COOH}$ groups results often in a red shift of the $\nu_{(\mathrm{C}=\mathrm{O})}$ band, and a blue shift of the $\nu_{(\mathrm{C}-\mathrm{OH})}$ band [45]. However, an electron (back) donation to the $\mathrm{COOH}$ side groups may compensate the effect due to hydrogen bonding. In consequence, both bands shift to higher wavenumbers. Similar trends were also observed in kinetic experiments. For example, $\nu_{(\mathrm{C}=\mathrm{O})}$ and $\nu_{(\mathrm{C}-\mathrm{OH})}$ shift to high wavenumbers until their maximum values are reached (ca. $150 \mathrm{~s}$ after stepping to the final potential) [27]. Considering the larger $\Delta \nu_{(\mathrm{C}=\mathrm{O})}$ and $\Delta \nu_{(\mathrm{C}-\mathrm{OH})}$ values of TMA compared with IA, we conclude that such an "indirect" electron (back) donation to the $\mathrm{COOH}$ moiety appears to be favored by a more stable hydrogen-bonded network. 


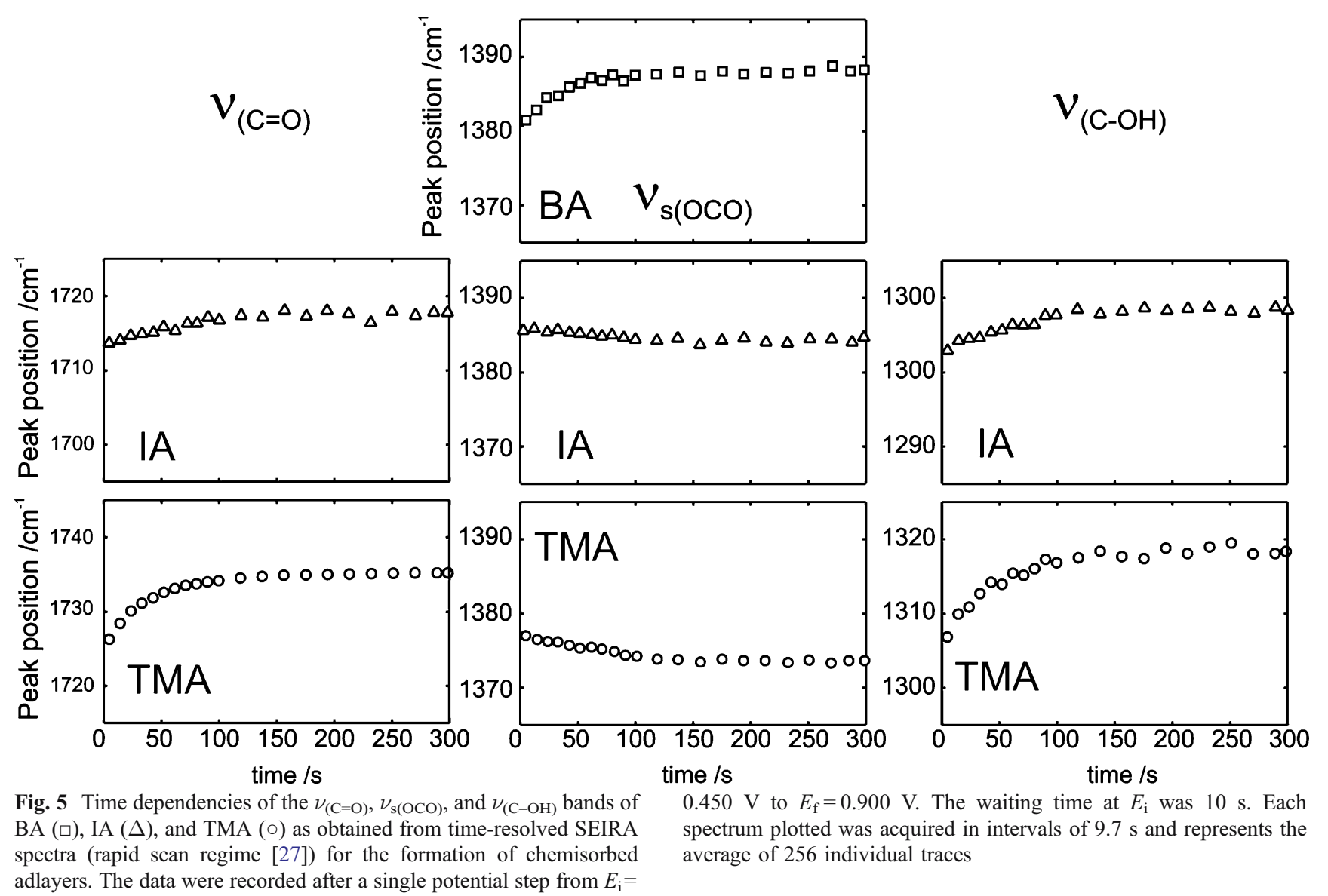

\section{Conclusions}

We have investigated the adsorption and self-assembly of BA, IA, and TMA on Au(111)- $(1 \times 1)$ single crystals and $\mathrm{Au}(111-25 \mathrm{~nm})$ quasi-single crystalline film electrodes in $0.1 \mathrm{M} \mathrm{HClO}_{4}$ by combining cyclic voltammetry with in situ ATR-SEIRAS and EC STM experiments. The adlayer structure can be tuned by changing the electrode potential. All three acids are physisorbed on the electrode surface in a planar orientation at negative charge densities (regions I and II), and chemisorbed in a tilted or perpendicular orientation at more positive potentials, which correspond to positive charge densities (region IV). Each individual molecule is coordinated onto the surface via one $\mathrm{COO}^{-}$group. In both physisorbed and chemisorbed adlayers, the acid molecules are connected to each other via intermolecular hydrogen bonds between adjacent undissociated $\mathrm{COOH}$ groups. The adlayers show different structure patterns depending on the change of lateral interactions and the strength of adsorbate-substrate coupling. The structure models and dimensions of the respective unit cells are summarized in Scheme 1 and Table 1.

A detailed analysis of the potential and time dependencies of the carboxyl and of the carboxylate vibration modes provides a comprehensive understanding of the steady-state structures and dynamic changes as triggered by the electrode potential. The lateral interactions (dipole-dipole coupling, $\pi$-stacking, or hydrogen binding) and the adsorbate-substrate coupling (electron (back) donation) determine the structure. They exhibit characteristic potential dependencies. In the chemisorbed adlayers, the strength of lateral interactions increases with an increasing number of $\mathrm{COOH}$ groups following the sequence $\mathrm{BA}<\mathrm{IA}<\mathrm{TMA}$. The adsorbate-substrate coupling is indicated by the electron back donation from the electrode to the $\mathrm{COO}^{-}$group and by an indirect electron donation to the $\mathrm{COOH}$ side-groups.

The combination of electrochemical methods, in situ STM, and SEIRAS reveals details on the potential-induced assemblies of BA, IA, and TMA monolayers on electrified gold/electrolyte interfaces. The universal role of the electrode potential in tuning the properties of the selfassembled patterns is demonstrated.

Acknowledgements The work was supported by the Volkswagen foundation under grant No. I80-879, IFMIT and the Research Center Jülich. The authors acknowledge the skilful help of U. Linke and of H. J. Bierfeld in preparing the gold single crystals and gold film electrodes. 


\section{References}

1. Bailey M, Brown CJ (1967) Acta Crystallogr B 22:387

2. Alcala R, Martinez-Carrera S (1972) Acta Crystallogr B 25:1671

3. De Feyter S, Gesquiere A, Abdel-Mottaleb MM, Grim PCM, De Shryver FC, Meiners C, Sieffert M, Valiyaveettil S, Müllen K (2000) Acc Chem Res 33:520

4. Melendres R, Hamilton AD (1998) Top Curr Chem 197:97

5. Barth JV, Costantini G, Kern K (2005) Nature 437:671

6. Herbstein FH (1996) In: Atwood JL, MacNico DD, Vögtle DD, Lehn JM (eds) Comprehenive supramolecular chemistry, vol 6. Pergamon, New York, pp 61

7. Kolotuchin SV, Thiessen PA, Fenlon EE, Wilson SR, Loweth CJ, Zimmerman SC (1999) Chem Eur J 5(9):2537

8. Chatterjee S, Pedireddi VR, Ranganathan A, Rao CNR (2000) J Mol Structure 520:107

9. Barth JV, Wechesser J, Lin N, Dmitriev A, Kern K (2003) Appl Phys A 76:645

10. Ermer O, Neudörfl J (2001) Chem Eur J 7:4961

11. Dai JC, Hu SM, Wu XT, Fu ZY, Du WX, Zhang HH, Sun RQ (2003) New J Chem 27:94

12. Melendez RE, Shrama CVK, Zaworotko MJ, Bauer C, Rogers RD (1996) Angew Chem Int Ed Engl 35:2213

13. Lin N, Dmitriev A, Weckesser J, Barth JV, Kern K (2002) Angew Chem Int Ed Engl 41:4779

14. Messina P, Dmitriev A, Lin N, Spillmann H, Abel M, Barth JV, Kern K (2002) J Am Chem Soc 124:14000

15. Ishikawa Y, Ohira A, Sakata M, Hirayama C, Kunitake MJ (2002) Chem Soc Chem Commun 2652

16. Su GJ, Zhang HM, Wan LJ, Bai CL, Wandlowski T (2004) J Phys Chem B 108:1931

17. Ikezawa Y, Sekiguchi R, Kitazume T (2000) Electrochim Acta 46:731

18. Li HQ, Roscoe SG, Lipkowski J (1999) J Electroanal Chem 478:67

19. Ikezawa Y, Yoshida A, Sekiguchi R (2000) Electrochim Acta 46:769
20. Lee MW, Kim MS, Kim K (1997) J Mol Struct 415:93

21. Dretschkow T, Wandlowski T (2003) Top Appl Phys 85:259

22. Schultz ZD, Gewirth AA (2005) Anal Chem 77:7373

23. Wandlowski T, Ataka K, Pronkin S, Diesing D (2004) Electrochim Acta 49:1233

24. Pronkin S, Wandlowski T (2003) J Electroanal Chem 550-551:131

25. Li HQ, Roscoe SG, Lipkowski J (2000) J Solution Chem 29:987

26. Osawa M, Ikeda M (1991) J Phys Chem 95:9914

27. Han B, Li Z, Pronkin S, Wandlowski T (2004) Can J Chem 82 (10): 1481

28. Li Z, Han B, Wandlowski T (2005) Langmuir 21:6915

29. Li Z, Han B, Wandlowski T (2007) (in preparation)

30. de Feyter S, Gesquiere A, Klapper M, Müllen K, Schryver FC (2003) Nano Lett 3:1485

31. Arenas JF, Marcos JI (1979) Spectrochim Acta 35A:355

32. Gonzalez-Sanchez F (1957) Spectrochim Acta 12:17

33. Lide DR (ed) (2001) In: Handbook of chemistry and physics, 82nd edn. CRC Press, Boca Raton, pp 8-45

34. Dean JA (1985) In: Lange's handbook of chemistry, 13th edn. McGraw-Hill, pp 5-42

35. Osawa M (1997) Bull Chem Soc Jpn 70:2861

36. Osawa M (2002) In: Chalmers JM, Griffiths PR (eds) Handbook of vibrational spectroscopy, vol 1 . Theory and instrumentation. Wiley, Chichister, p 785

37. Nichols R (1992) In: Lipkowski J, Ross PN (eds) Adsorption of molecules at electrodes. VCH, New York, p 347

38. Lambert DK (1996) Electrochim Acta 41:623

39. Ashley K, Pons S (1988) Chem Rev 88:673

40. Ataka K, Osawa M (1999) J Electroanal Chem 460:188

41. Wandlowski T, Ataka K, Mayer D (2002) Langmuir 18:4331

42. Noda H, Wan LJ, Osawa M (2001) Phys Chem Chem Phys 3:3336

43. Kwon YJ, Son DH, Ahn SJ, Kim MS, Kim K (1994) J Phys Chem 98:8481

44. Kim SH, Ahn SJ, Kim K (1996) J Phys Chem 100:7174

45. Colthup NB, Daley LH, Wiberly SE (1990) Introduction to infrared and Raman spectroscopy. Academic Press, Boston 